\title{
On the 4-rank of tame kernels of quadratic number fields
}

\author{
by \\ Xuejun Guo (Nanjing)
}

1. Introduction and notations. Let $F$ be a quadratic number field, and $\mathcal{O}_{F}$ the ring of integers in $F$. For a finite abelian group $A$, let $r_{4}(A)=$ $\operatorname{dim}_{\mathbb{F}_{2}} A^{2} / A^{4}$ be the 4-rank of $A$. The purpose of this paper is to compute the density of real (resp. imaginary) quadratic number fields with $r_{4}\left(K_{2} \mathcal{O}_{F}\right)=r$ in the set of all real (resp. imaginary) quadratic number fields. First, we introduce the following definition of densities.

DeFinition 1.1. Let $D$ be a fundamental discriminant, i.e., the discriminant of some quadratic number field. Let

$$
g_{r}(D)= \begin{cases}1 & \text { if } r_{4}\left(K_{2} \mathcal{O}_{\mathbb{Q}(\sqrt{D})}\right)=r, \\ 0 & \text { otherwise. }\end{cases}
$$

Then the density of real quadratic number fields $F$ with $r_{4}\left(K_{2} \mathcal{O}_{F}\right)=r$ in the set of all real quadratic number fields is

$$
d_{r}^{+}=\lim _{x \rightarrow \infty} \frac{\sum_{0<D<x} g_{r}(D)}{\sum_{0<D<x} 1}
$$

and the density of imaginary quadratic number fields $E$ with $r_{4}\left(K_{2} \mathcal{O}_{E}\right)=r$ in the set of all imaginary quadratic number fields is

$$
d_{r}^{-}=\lim _{x \rightarrow \infty} \frac{\sum_{0<-D<x} g_{r}(D)}{\sum_{0<-D<x} 1} .
$$

The study of the 4-ranks of tame kernels of quadratic number fields has a long history. One can find formulae for the 4-rank of $K_{2} \mathcal{O}_{F}$ in [3] and [7]. In 1995, Qin [11], [12] gave an efficient method of computing the 4-ranks of $K_{2} \mathcal{O}_{F}$. Later Hurrelbrink and Kolster [6] and Qin [13] gave improved methods of computing the 4-ranks. 
The densities of 4-ranks of tame kernels of quadratic number fields have been studied in several papers. In [8]-[9], [2], Osburn and Cheng studied the densities of real (resp. imaginary) quadratic number fields with $r_{4}\left(K_{2} \mathcal{O}_{F}\right)=r$ in the set of all real (resp. imaginary) quadratic number fields with exactly $t$ ramified primes, where $t$ is an integer not greater than 3 . In 2007, Yin [14], [15] gave the tables of the 4-rank of $K_{2} \mathcal{O}_{F}$, assuming the number of odd prime factors of the discriminant of $F$ is not greater than 4. In [17], Yue and Yu computed the limiting densities of real (resp. imaginary) quadratic number fields with $r_{4}\left(K_{2} \mathcal{O}_{F}\right)=r$ in the set of all real (resp. imaginary) quadratic number fields with exactly $t$ ramified primes, as $t \rightarrow \infty$.

In Definition 1.1, the quadratic number fields are ordered by the size of their discriminants. Since every quadratic number field can be written as $\mathbb{Q}(\sqrt{n})$, where $n$ is a square free integer, we can also order them by the size of $n$. This observation leads to the following alternative definition of the densities.

Definition 1.2. Let $D$ be a fundamental discriminant and

$$
\sigma(D)= \begin{cases}D & \text { if } D \equiv 1 \bmod 4 \\ D / 4 & \text { if } D \equiv 0 \bmod 4\end{cases}
$$

Then the density of real quadratic number fields $F$ with $r_{4}\left(K_{2} \mathcal{O}_{F}\right)=r$ in the set of all real quadratic number fields is

$$
d_{r}=\lim _{x \rightarrow \infty} \frac{\sum_{0<\sigma(D)<x} g_{r}(D)}{\sum_{0<\sigma(D)<x} 1},
$$

and the density of imaginary quadratic number fields $E$ with $r_{4}\left(K_{2} \mathcal{O}_{E}\right)=r$ in the set of all imaginary quadratic number fields is

$$
d_{r}^{\prime}=\lim _{x \rightarrow \infty} \frac{\sum_{0<-\sigma(D)<x} g_{r}(D)}{\sum_{0<-\sigma(D)<x} 1} .
$$

We will prove in Section 4 that $d_{r}^{+}=d_{r}$ and $d_{r}^{-}=d_{r}^{\prime}$. A direct corollary is that the real (imaginary) quadratic number fields with $r_{4}\left(K_{2} \mathcal{O}_{F}\right)=r$ distribute equally in different residue classes of $\sigma(D)$ modulo 4. The main result of this paper is the following theorem.

Theorem 1.3. Let $r$ be an integer. Then

$$
\begin{array}{ll}
d_{0}^{+}=0, \\
d_{r}^{+}=\frac{2^{-r(r-1)} \prod_{k=1}^{\infty}\left(1-2^{-k}\right)}{\left(1-2^{-r}\right) \prod_{k=1}^{r-1}\left(1-2^{-k}\right)^{2}} & \text { for } r \geq 1, \\
d_{r}^{-}=2^{-r^{2}} \frac{\prod_{k=1}^{\infty}\left(1-2^{-k}\right)}{\prod_{k=1}^{r}\left(1-2^{-k}\right)^{2}} & \text { for } r \geq 0 .
\end{array}
$$


Let us briefly review Yue and Yu's results in [17]. Let $n$ be a square free positive integer, $x$ a positive real number, $r$ a nonnegative integer, and $t$ a positive integer. We define

$$
\begin{aligned}
A_{t} & =\{F=\mathbb{Q}(\sqrt{n}) \mid \text { exactly } t \text { primes ramify in } E=\mathbb{Q}(\sqrt{-n}) / \mathbb{Q}\}, \\
A_{t ; x} & =\left\{F=\mathbb{Q}(\sqrt{n}) \in A_{t} \mid n \leq x\right\}, \\
\bar{A}_{t, r ; x} & =\left\{F=\mathbb{Q}(\sqrt{n}) \in A_{t ; x} \mid r_{4}\left(K_{2} \mathcal{O}_{F}\right)=r\right\}, \\
\bar{d}_{t, r} & =\lim _{x \rightarrow \infty} \frac{\left|\bar{A}_{t, r ; x}\right|}{\left|A_{t ; x}\right|}, \quad \bar{d}_{\infty, r}=\lim _{t \rightarrow \infty} d_{t, r} .
\end{aligned}
$$

Similarly, for imaginary quadratic fields, we define

$$
\begin{aligned}
B_{t} & =\{E=\mathbb{Q}(\sqrt{-n}) \mid \text { exactly } t \text { primes ramify in } F=\mathbb{Q}(\sqrt{n}) / \mathbb{Q}\}, \\
B_{t ; x} & =\left\{E=\mathbb{Q}(\sqrt{-n}) \in B_{t} \mid n \leq x\right\}, \\
\bar{B}_{t, r ; x} & =\left\{E=\mathbb{Q}(\sqrt{-n}) \in B_{t ; x} \mid r_{4}\left(K_{2} \mathcal{O}_{E}\right)=r\right\}, \\
\bar{d}_{t, r}^{\prime} & =\lim _{x \rightarrow \infty} \frac{\left|\bar{B}_{t, r ; x}\right|}{\left|B_{t ; x}\right|}, \quad \bar{d}_{\infty, r}^{\prime}=\lim _{t \rightarrow \infty} d_{t, r} .
\end{aligned}
$$

The main result of [17] is the following theorem.

Theorem 1.4 ([17, Theorems 3.1 and 3.2]). Let $r$ be an integer. Then

$$
\begin{array}{ll}
\bar{d}_{\infty, 0}=0, \\
\bar{d}_{\infty, r}=\frac{2^{-r(r-1)} \prod_{k=1}^{\infty}\left(1-2^{-k}\right)}{\left(1-2^{-r}\right) \prod_{k=1}^{r-1}\left(1-2^{-k}\right)^{2}} & \text { for } r \geq 1, \\
\bar{d}_{\infty, r}^{\prime}=2^{-r^{2}} \frac{\prod_{k=1}^{\infty}\left(1-2^{-k}\right)}{\prod_{k=1}^{r}\left(1-2^{-k}\right)^{2}} & \text { for } r \geq 0 .
\end{array}
$$

By comparing Theorem 1.3 with Theorem 1.4, one can see that

$$
d_{r}^{+}=\bar{d}_{\infty, r}, \quad d_{r}^{-}=\bar{d}_{\infty, r}^{\prime} .
$$

This is not accidental. It is just what happened in [4]. If you compare Theorem 3 of [4] with the formulae (1.5) and (1.6) of [5], you can see the analogous accordance. I would like to mention that Theorems 3.1 and 3.2 of [17] gave a strong support for Theorem 1.3 above.

Sometimes it is more interesting to consider $\bar{d}_{t, r}$ and $\bar{d}_{t, r}^{\prime}$ than $d_{r}^{+}$and $d_{r}^{-}$. The reason is that some arithmetic invariants which appear in computing $\bar{d}_{t, r}$ and $\bar{d}_{t, r}^{\prime}$ will disappear if one considers the natural densities $d_{r}^{+}$and $d_{r}^{-}$. If one considers more general number fields, e.g., the cubic cyclic number fields, the Brauer-Manin obstruction will be involved in the computation. We will consider this problem in another paper.

In the last section, some numerical evidence is discussed. 
2. The 4-rank of tame kernels of real quadratic number fields. Let $A_{t}, A_{t ; x}, \bar{A}_{t, r ; x}, \bar{d}_{t, r}, \bar{d}_{\infty, r}$ be as in Section 1. We denote the narrow class group of $F$ by $C(F)$. Let $n>2$ be a square free integer, and

$$
\begin{aligned}
A_{t, r} & =\left\{F \in A_{t} \mid r_{4}(C(E))=r\right\}, \quad A_{t, r ; x}=\left\{F \in A_{t, r} \mid n \leq x\right\}, \\
\bar{d}_{r}(j) & =\lim _{x \rightarrow \infty} \frac{\sum_{t=1}^{\infty}\left|A_{t, r+j ; x} \cap \bar{A}_{t, r ; x}\right|}{\sum_{t=1}^{\infty}\left|A_{t ; x}\right|}, \quad j=1,0, \text { or }-1 .
\end{aligned}
$$

Note that for any $x$, there are only finitely many terms in $\sum_{t=1}^{\infty}\left|A_{t ; x}\right|$.

Let $N(m, k, r)$ be the number of $m \times m$ matrices $M=\left(a_{i j}\right)$, over $F_{2}$ with the following properties:

(i) $a_{i j} \neq a_{j i}$ for $1 \leq i<j \leq k$,

(ii) $a_{i j}=a_{j i}$ when $k+1 \leq i \leq m$ and $1 \leq j \leq m$,

(iii) $\operatorname{rank} M=r$.

Let

$$
d_{t, r ; x}=\frac{\left|A_{t, r ; x}\right|}{\left|A_{t ; x}\right|}, \quad d_{t, r}=\lim _{x \rightarrow \infty} d_{t, r ; x}, \quad d_{\infty, r}=\lim _{t \rightarrow \infty} d_{t, r} .
$$

Then by Proposition 2.1 of [5],

$$
\begin{aligned}
d_{t, r} & =\sum_{\substack{1 \leq l \leq t \\
l \text { odd }}} N(t-1, l-1, t-1-r)\left(\begin{array}{l}
t \\
l
\end{array}\right) 2^{1-\left(t^{2}+t\right) / 2}, \\
d_{\infty, r} & =\frac{2^{-r^{2}} \prod_{k=1}^{\infty}\left(1-2^{-k}\right)}{\prod_{k=1}^{r}\left(1-2^{-k}\right)^{2}} .
\end{aligned}
$$

Let $F=\mathbb{Q}(\sqrt{D})$ and

$$
\begin{aligned}
& S^{+}(x, k, a, b):=\sum_{\substack{0<D<x \\
D \equiv a \bmod b}} 2^{k \mathrm{rk}_{4}(C(F))}, \\
& S^{-}(x, k, a, b):=\sum_{\substack{0<-D<x \\
-D \equiv a \bmod b}} 2^{k \operatorname{rk}_{4}(C(F))} .
\end{aligned}
$$

Lemma 2.1. Let $D$ be a fundamental discriminant, $r$ a nonnegative integer, $n$ a square free integer, and $F=\mathbb{Q}(\sqrt{D})$. Let

$$
f_{r}(D)= \begin{cases}1 & \text { if } r_{4}(C(F))=r, \\ 0 & \text { otherwise }\end{cases}
$$

and $\sigma(D)$ be as in Definition 1.2. Then
(1) $\lim _{x \rightarrow \infty} \frac{\sum_{0<D<x} f_{r}(D)}{\sum_{0<D<x} 1}=\lim _{x \rightarrow \infty} \frac{\sum_{0<\sigma(D)<x} f_{r}(D)}{\sum_{0<\sigma(D)<x} 1}$,
(2) $\lim _{x \rightarrow \infty} \frac{\sum_{0<-D<x} f_{r}(D)}{\sum_{0<-D<x} 1}=\lim _{x \rightarrow \infty} \frac{\sum_{0<-\sigma(D)<x} f_{r}(D)}{\sum_{0<-\sigma(D)<x} 1}$. 
Proof. (1) Let

$$
\begin{aligned}
S_{1 ; x} & =\sum_{\substack{0<D<x \\
D \equiv 1 \bmod 4}} 1, \quad T_{1, r ; x}=\sum_{\substack{0<D<x \\
D \equiv 1 \bmod 4}} f_{r}(D), \\
S_{2 ; x} & =\sum_{\substack{0<D<4 x \\
D \equiv 0 \bmod 8}} 1, \quad T_{2, r ; x}=\sum_{\substack{0<D<4 x \\
D \equiv 0 \bmod 8}} f_{r}(D), \\
S_{3 ; x}= & \sum_{\substack{0<D<4 x \\
D \equiv 4 \bmod 8}} 1, \quad T_{3, r ; x}=\sum_{\substack{0<D<4 x \\
D \equiv 4 \bmod 8}} f_{r}(D) .
\end{aligned}
$$

For a fixed $x$, let $R_{x}$ be an integer large enough, and

$$
a_{r ; x}=T_{1, r ; x}, \quad b_{r ; x}=T_{2, r ; x}, \quad c_{r ; x}=T_{3, r ; x}, \quad 0 \leq r \leq R_{x} .
$$

Then we have the following system of linear equations:

$$
\begin{aligned}
& \sum_{j=0}^{R_{x}} 2^{-i j} a_{j ; x}=S^{+}(x, i, 1,4), \\
& \sum_{j=0}^{R_{x}} 2^{-i j} b_{j ; x}=S^{+}(4 x, i, 0,8), \\
& \sum_{j=0}^{R_{x}} 2^{-i j} c_{j ; x}=S^{+}(4 x, i, 4,8),
\end{aligned}
$$

where $i=0,1, \ldots, R_{x}$. If we consider all $a_{j ; x}$ as indeterminates, then the matrices of coefficients are Vandermonde matrices.

By equations (5), (7), and (9) of [4],

$$
S^{+}(x, i, 1,4) \sim S^{+}(4 x, i, 0,8) \sim S^{+}(4 x, i, 4,8) \quad \text { as } x \rightarrow \infty .
$$

Hence by solving (2.1), (2.2) and (2.3), we have

$$
a_{r ; x} \sim b_{r ; x} \sim c_{r ; x} \quad \text { as } x \rightarrow \infty,
$$

for any fixed $r$. By $[4,(16)]$,

$$
S_{1 ; x} \sim S_{2 ; x} \sim S_{3 ; x} \sim \frac{2}{\pi^{2}} x \quad \text { as } x \rightarrow \infty .
$$

So if $x$ is large enough, we have

$$
\frac{a_{r ; x}}{S_{1 ; x}} \approx \frac{b_{r ; x}}{S_{2 ; x}} \approx \frac{c_{r ; x}}{S_{3 ; x}} .
$$


Hence

$$
\begin{aligned}
\lim _{x \rightarrow \infty} \frac{\sum_{0<\sigma(D)<x} f_{r}(D)}{\sum_{0<\sigma(D)<x} 1} & =\lim _{x \rightarrow \infty} \frac{a_{r ; x}+b_{r ; x}+c_{r ; x}}{S_{1 ; x}+S_{2 ; x}+S_{3 ; x}} \\
& =\lim _{x \rightarrow \infty} \frac{a_{r ; x}}{S_{1 ; x}}=\lim _{x \rightarrow \infty} \frac{b_{r ; x / 4}}{S_{2 ; x / 4}}=\lim _{x \rightarrow \infty} \frac{c_{r ; x / 4}}{S_{3 ; x / 4}} \\
& =\lim _{x \rightarrow \infty} \frac{a_{r ; x}+b_{r ; x / 4}+c_{r ; x / 4}}{S_{1 ; x}+S_{2 ; x / 4}+S_{3 ; x / 4}} \\
& =\lim _{x \rightarrow \infty} \frac{\sum_{0<D<x} f_{r}(D)}{\sum_{0<D<x} 1} .
\end{aligned}
$$

(2) can be proved by analogous arguments.

Let $D, r, g_{r}(D), d_{r}^{+}, d_{r}^{-}$be as in the introduction.

THEOREM 2.2. With the above notations,

$$
\begin{aligned}
& \text { (1) } \bar{d}_{r}(1)=0 \\
& \text { for } r \geq 0 \text {, } \\
& \text { (2) } \quad \bar{d}_{r}(0)=\left(1-2^{-r}\right) \frac{2^{-r^{2}} \prod_{k=1}^{\infty}\left(1-2^{-k}\right)}{\prod_{k=1}^{r}\left(1-2^{-k}\right)^{2}} \quad \text { for } r \geq 0 \text {, } \\
& \text { (3) } \quad \bar{d}_{r}(-1)=2^{-r^{2}+r} \frac{\prod_{k=1}^{\infty}\left(1-2^{-k}\right)}{\prod_{k=1}^{r-1}\left(1-2^{-k}\right)^{2}} \quad \text { for } r \geq 1 \text {. }
\end{aligned}
$$

Proof. (1) Let

$$
\alpha_{t, r ; x}=\frac{\left|A_{t, r ; x} \cap \bar{A}_{t, r-1 ; x}\right|}{\left|A_{t, r ; x}\right|}, \quad \alpha_{t, r}=\lim _{x \rightarrow \infty} \alpha_{t, r ; x}, \quad \alpha_{\infty, r}=\lim _{t \rightarrow \infty} \alpha_{t, r} .
$$

By Theorem 3.1 of [16], $F \in A_{t, r ; x} \cap \bar{A}_{t, r-1 ; x}$ only if 2 is the norm of some element in $F$, i.e., all primes which are ramified in $F$ are congruent to 1 or -1 modulo 8 . Since all primes are equally distributed among all residue classes modulo 8 , we have

$$
\alpha_{t, r} \leq 2^{-t} \text {. }
$$

By Theorem 3.2 of [16], there are other necessary conditions for

$$
F \in A_{t, r ; x} \cap \bar{A}_{t, r-1 ; x},
$$

besides $2 \in \operatorname{Norm}_{F / \mathbb{Q}}\left(F^{*}\right)$. Hence $\alpha_{t, r}$ is strictly less than $2^{-t}$.

Let $N_{x}$ be the number of square free integers up to $x$ with exactly $t$ prime factors. By $[5,(2.4)]$,

$$
N_{x} \sim \frac{1}{(t-1) !} \frac{x(\log \log x)^{t-1}}{\log x} \quad(\text { as } x \rightarrow \infty) .
$$

So for a fixed $T$, if $x$ is large enough, then

$$
\frac{\sum_{t=1}^{T-1}\left|A_{t ; x}\right|}{\left|A_{T ; x}\right|}<\frac{T !}{\log \log x}
$$


can be as small as we wish. Hence

$$
\begin{aligned}
\bar{d}_{r}(1) & =\lim _{x \rightarrow \infty} \frac{\sum_{t=1}^{\infty}\left|A_{t, r+1 ; x} \cap \bar{A}_{t, r ; x}\right|}{\sum_{t=1}^{\infty}\left|A_{t ; x}\right|}=\lim _{x \rightarrow \infty} \frac{\sum_{t=1}^{\infty} \alpha_{t, r+1 ; x}\left|A_{t, r+1 ; x}\right|}{\sum_{t=1}^{\infty}\left|A_{t ; x}\right|} \\
& =\lim _{x \rightarrow \infty} \frac{\sum_{t=T}^{\infty} \alpha_{t, r+1 ; x}\left|A_{t, r+1 ; x}\right|}{\sum_{t=T}^{\infty}\left|A_{t ; x}\right|} \quad \text { for any fixed } T \\
& \leq \lim _{x \rightarrow \infty} \frac{2^{-T} \sum_{t=T}^{\infty}\left|A_{t, r+1 ; x}\right|}{\sum_{t=T}^{\infty}\left|A_{t ; x}\right|} \leq 2^{-T},
\end{aligned}
$$

which implies $\bar{d}_{r}(1)=0$.

(2) Let

$$
\beta_{t, r ; x}=\frac{\left|A_{t, r ; x} \cap \bar{A}_{t, r ; x}\right|}{\left|A_{t, r ; x}\right|}, \quad \beta_{t, r}=\lim _{x \rightarrow \infty} \beta_{t, r ; x}, \quad \beta_{\infty, r}=\lim _{t \rightarrow \infty} \beta_{t, r} .
$$

By similar arguments to the proof of (1), we have

$$
\begin{aligned}
\bar{d}_{r}(0) & =\lim _{x \rightarrow \infty} \frac{\sum_{t=1}^{\infty}\left|A_{t, r ; x} \cap \bar{A}_{t, r ; x}\right|}{\sum_{t=1}^{\infty}\left|A_{t ; x}\right|}=\lim _{x \rightarrow \infty} \frac{\sum_{t=1}^{\infty} \beta_{t, r ; x}\left|A_{t, r ; x}\right|}{\sum_{t=1}^{\infty}\left|A_{t ; x}\right|} \\
& =\beta_{\infty, r} \lim _{x \rightarrow \infty} \frac{\sum_{t=1}^{\infty}\left|A_{t, r ; x}\right|}{\sum_{t=1}^{\infty}\left|A_{t ; x}\right|} .
\end{aligned}
$$

By $[17,(3.19)]$,

$$
\beta_{t, r}=1-\frac{2^{t-1-r}-1}{2^{t-1}-1}, \quad \beta_{\infty, r}=1-2^{-r} .
$$

By Theorem 3 of [4] and Lemma 2.1,

$$
\lim _{x \rightarrow \infty} \frac{\sum_{t=1}^{\infty}\left|A_{t, r ; x}\right|}{\sum_{t=1}^{\infty}\left|A_{t ; x}\right|}=\frac{2^{-r^{2}} \prod_{k=1}^{\infty}\left(1-2^{-k}\right)}{\prod_{k=1}^{r}\left(1-2^{-k}\right)^{2}} .
$$

Hence

$$
\bar{d}_{r}(0)=\left(1-2^{-r}\right) \frac{2^{-r^{2}} \prod_{k=1}^{\infty}\left(1-2^{-k}\right)}{\prod_{k=1}^{r}\left(1-2^{-k}\right)^{2}} .
$$

(3) Let $r$ be a positive integer, and

$$
\gamma_{t, r-1 ; x}=\frac{\left|A_{t, r-1 ; x} \cap \bar{A}_{t, r ; x}\right|}{\left|A_{t, r-1 ; x}\right|}, \quad \gamma_{t, r}=\lim _{x \rightarrow \infty} \gamma_{t, r ; x}, \quad \gamma_{\infty, r}=\lim _{t \rightarrow \infty} \gamma_{t, r} .
$$

By similar arguments, we have

$$
\begin{aligned}
\bar{d}_{r}(-1) & =\lim _{x \rightarrow \infty} \frac{\sum_{t=1}^{\infty}\left|A_{t, r-1 ; x} \cap \bar{A}_{t, r ; x}\right|}{\sum_{t=1}^{\infty}\left|A_{t ; x}\right|} \\
& =\lim _{x \rightarrow \infty} \frac{\sum_{t=1}^{\infty} \gamma_{t, r-1 ; x}\left|A_{t, r-1 ; x}\right|}{\sum_{t=1}^{\infty}\left|A_{t ; x}\right|} \\
& =\gamma_{\infty, r-1} \lim _{x \rightarrow \infty} \frac{\sum_{t=1}^{\infty}\left|A_{t, r-1 ; x}\right|}{\sum_{t=1}^{\infty}\left|A_{t ; x}\right|} .
\end{aligned}
$$


By $[17,(3.19)]$,

$$
\gamma_{\infty, r-1}=2^{-r+1}
$$

By Theorem 3 of [4] and Lemma 2.1,

$$
\lim _{x \rightarrow \infty} \frac{\sum_{t=1}^{\infty}\left|A_{t, r-1 ; x}\right|}{\sum_{t=1}^{\infty}\left|A_{t ; x}\right|}=\frac{2^{-(r-1)^{2}} \prod_{k=1}^{\infty}\left(1-2^{-k}\right)}{\prod_{k=1}^{r-1}\left(1-2^{-k}\right)^{2}} .
$$

Hence

$$
\bar{d}_{r}(-1)=2^{-r^{2}+r} \frac{\prod_{k=1}^{\infty}\left(1-2^{-k}\right)}{\prod_{k=1}^{r-1}\left(1-2^{-k}\right)^{2}} .
$$

TheOREM 2.3. Let $r$ be an integer. Then the density of real quadratic number fields $F$ with $r_{4}\left(K_{2} \mathcal{O}_{F}\right)=r$ in the set of all real quadratic number fields is

$$
\begin{aligned}
& d_{0}=0, \\
& d_{r}=\frac{2^{-r(r-1)} \prod_{k=1}^{\infty}\left(1-2^{-k}\right)}{\left(1-2^{-r}\right) \prod_{k=1}^{r-1}\left(1-2^{-k}\right)^{2}} \quad \text { for } r \geq 1 .
\end{aligned}
$$

Proof. By Theorems 3.1 and 4.1 of [16], we know that

$$
d_{r}= \begin{cases}\bar{d}_{r}(0)+\bar{d}_{r}(1) & \text { for } r=0, \\ \bar{d}_{r}(-1)+\bar{d}_{r}(0)+\bar{d}_{r}(1) & \text { for } r \geq 1 .\end{cases}
$$

Hence we get the result by Theorem 2.2 .

3. The 4-rank of tame kernels of imaginary quadratic number fields. Let $B_{t}, B_{t ; x}, \bar{B}_{t, r ; x}, \bar{d}_{t, r}^{\prime}, \bar{d}_{\infty, r}^{\prime}$ be as in the introduction, and $n>2$ be a square free integer. Let $F=\mathbb{Q}(\sqrt{n})$ and $E=\mathbb{Q}(\sqrt{-n})$, and

$$
\begin{aligned}
B_{t, r} & =\left\{E \in B_{t} \mid r_{4}(C(F))=r\right\}, \quad B_{t, r ; x}=\left\{E \in B_{t, r} \mid n \leq x\right\}, \\
\bar{d}_{r}^{\prime}(j) & =\lim _{x \rightarrow \infty} \frac{\sum_{t=1}^{\infty}\left|B_{t, r+j ; x} \cap \bar{B}_{t, r ; x}\right|}{\sum_{t=1}^{\infty}\left|B_{t ; x}\right|}, \quad j=1,0,-1, \\
d_{t, r ; x}^{\prime} & =\frac{\left|B_{t, r ; x}\right|}{\left|B_{t ; x}\right|}, \quad d_{t, r}^{\prime}=\lim _{x \rightarrow \infty} d_{t, r ; x}^{\prime}, \quad d_{\infty, r}^{\prime}=\lim _{t \rightarrow \infty} d_{t, r}^{\prime} .
\end{aligned}
$$

Theorem 3.1. With the above notations,

(1) $\bar{d}_{r}^{\prime}(1)=0$ for $r \geq 0$,

(2) $\quad \bar{d}_{r}^{\prime}(0)=\left(1-2^{-r-1}\right) \frac{2^{-r^{2}-r} \prod_{k=1}^{\infty}\left(1-2^{-k}\right)}{\prod_{k=1}^{r}\left(1-2^{-k}\right) \prod_{k=1}^{r+1}\left(1-2^{-k}\right)} \quad$ for $r \geq 0$,

(3) $\bar{d}_{r}^{\prime}(-1)=2^{-r^{2}}$

$$
\frac{\prod_{k=1}^{\infty}\left(1-2^{-k}\right)}{\prod_{k=1}^{r-1}\left(1-2^{-k}\right) \prod_{k=1}^{r}\left(1-2^{-k}\right)} \quad \text { for } r \geq 1 .
$$

Proof. (1) By Theorem 4.1 of $[16], 2 \in \operatorname{Norm}_{E / \mathbb{Q}}\left(E^{*}\right)$ is a necessary condition for

$$
r_{4}\left(K_{2}\left(\mathcal{O}_{E}\right)\right)+1=r_{4}(C(F)) .
$$


Since 2 is a norm of some element in $E=\mathbb{Q}(\sqrt{-n})$ if and only if all the prime factors of $n$ are congruent to 1 or -1 modulo 8 , the density of theses fields will be 0 as $t \rightarrow \infty$. Hence (1) holds.

(2) Let

$$
\beta_{t, r ; x}^{\prime}=\frac{\left|B_{t, r ; x} \cap \bar{B}_{t, r ; x}\right|}{\left|B_{t, r ; x}\right|}, \quad \beta_{t, r}^{\prime}=\lim _{x \rightarrow \infty} \beta_{t, r ; x}, \quad \beta_{\infty, r}^{\prime}=\lim _{t \rightarrow \infty} \beta_{t, r} .
$$

All the imaginary quadratic number fields $\mathbb{Q}(\sqrt{-n}) \in B_{t}$ can be divided into three types:

$$
\begin{aligned}
& n=p_{1} \cdots p_{t} \quad \text { with an even number of } p_{i} \equiv 3 \bmod 4 \text {, or } \\
& n=p_{1} \cdots p_{t-1} \quad \text { with an odd number of } p_{i} \equiv 3 \bmod 4 \text {, or } \\
& \text { (3.3) } n=2 p_{1} \cdots p_{t-1} \text {. }
\end{aligned}
$$

Let

$$
B_{t, x}^{(i)}=\{E=\mathbb{Q}(\sqrt{-n}) \mid n \text { satisfies }(3 . i)\}, \quad i=1,2,3 .
$$

Then

$$
\begin{aligned}
\left|B_{t, x}^{(1)}\right| \sim\left|B_{t, x}\right| & \text { as } x \rightarrow \infty, \\
\left|B_{t, x}^{(i)}\right|=o\left(\left|B_{t, x}\right|\right) & \text { as } x \rightarrow \infty, \text { for } i=2,3 .
\end{aligned}
$$

Hence we confine our attention to those $n$ which satisfy (3.1).

If $n$ satisfies (3.1), then $n \equiv 1 \bmod 4$. Furthermore, if $n \equiv 5 \bmod 8$, then

$$
r_{4}\left(K_{2} \mathcal{O}_{E}\right)=r_{4}(C(F))
$$

by Theorem 4.1 of [16]. Next we will consider the case $n \equiv 1 \bmod 8$. Let

$$
\begin{aligned}
& S_{t, r ; x}^{(1)}=\left\{E \in B_{t, r ; x} \cap \bar{B}_{t, r ; x} \mid n \equiv 1 \bmod 8 \text { and } 2 \notin \operatorname{Norm}_{E / \mathbb{Q}}\left(E^{*}\right)\right\}, \\
& T_{t, r ; x}^{(1)}=\left\{E \in B_{t, r ; x} \mid n \equiv 1 \bmod 8 \text { and } 2 \notin \operatorname{Norm}_{E / \mathbb{Q}}\left(E^{*}\right)\right\} .
\end{aligned}
$$

Then by $[17,(3.25)]$, we have

$$
\lim _{t \rightarrow \infty} \lim _{x \rightarrow \infty} \frac{\left|S_{t, r ; x}^{(1)}\right|}{\left|T_{t, r ; x}^{(1)}\right|}=1-2^{-r} .
$$

Since $n \equiv 1 \bmod 8$ and $n \equiv 5 \bmod 8$ are equally likely in $B_{t}$, we have

$$
\beta_{\infty, r}^{\prime}=\frac{1}{2}+\frac{1}{2}\left(1-2^{-r}\right)=1-2^{-r-1} .
$$

Hence by similar arguments to the proof of (1), we have

$$
\begin{aligned}
\bar{d}_{r}^{\prime}(0) & =\lim _{x \rightarrow \infty} \frac{\sum_{t=1}^{\infty}\left|B_{t, r ; x} \cap \bar{B}_{t, r ; x}\right|}{\sum_{t=1}^{\infty}\left|B_{t ; x}\right|}=\lim _{x \rightarrow \infty} \frac{\sum_{t=1}^{\infty} \beta_{t, r ; x}^{\prime}\left|B_{t, r ; x}\right|}{\sum_{t=1}^{\infty}\left|B_{t ; x}\right|} \\
& =\beta_{\infty, r}^{\prime} \lim _{x \rightarrow \infty} \frac{\sum_{t=1}^{\infty}\left|B_{t, r ; x}\right|}{\sum_{t=1}^{\infty}\left|B_{t ; x}\right|} .
\end{aligned}
$$


By Theorem 3 of [4] and Lemma 2.1,

Hence

$$
\lim _{x \rightarrow \infty} \frac{\sum_{t=1}^{\infty}\left|B_{t, r ; x}\right|}{\sum_{t=1}^{\infty}\left|B_{t ; x}\right|}=\frac{2^{-r(r+1)} \prod_{k=1}^{\infty}\left(1-2^{-k}\right)}{\prod_{k=1}^{r}\left(1-2^{-k}\right) \prod_{k=1}^{r+1}\left(1-2^{-k}\right)} .
$$

$$
\bar{d}_{r}^{\prime}(0)=\left(1-2^{-r-1}\right) \frac{2^{-r^{2}-r} \prod_{k=1}^{\infty}\left(1-2^{-k}\right)}{\prod_{k=1}^{r}\left(1-2^{-k}\right) \prod_{k=1}^{r+1}\left(1-2^{-k}\right)} .
$$

(3) Let $r$ be a positive integer, and

$$
\begin{aligned}
\gamma_{t, r-1 ; x}^{\prime} & =\frac{\left|B_{t, r-1 ; x} \cap \bar{B}_{t, r ; x}\right|}{\left|B_{t, r-1 ; x}\right|}, \\
\gamma_{t, r}^{\prime} & =\lim _{x \rightarrow \infty} \gamma_{t, r ; x}^{\prime}, \quad \gamma_{\infty, r}^{\prime}=\lim _{t \rightarrow \infty} \gamma_{t, r}^{\prime} .
\end{aligned}
$$

By similar arguments, we have

$$
\begin{aligned}
\bar{d}_{r}^{\prime}(-1) & =\lim _{x \rightarrow \infty} \frac{\sum_{t=1}^{\infty}\left|B_{t, r-1 ; x} \cap \bar{B}_{t, r ; x}\right|}{\sum_{t=1}^{\infty}\left|B_{t ; x}\right|}=\lim _{x \rightarrow \infty} \frac{\sum_{t=1}^{\infty} \gamma_{t, r-1 ; x}^{\prime}\left|B_{t, r-1 ; x}\right|}{\sum_{t=1}^{\infty}\left|B_{t ; x}\right|} \\
& =\gamma_{\infty, r-1}^{\prime} \lim _{x \rightarrow \infty} \frac{\sum_{t=1}^{\infty}\left|B_{t, r-1 ; x}\right|}{\sum_{t=1}^{\infty}\left|B_{t ; x}\right|} .
\end{aligned}
$$

In the proof of $(2)$, we have shown that if $n \equiv 1 \bmod 8$ and $2 \notin \operatorname{Norm}_{E / \mathbb{Q}}\left(E^{*}\right)$, then

$$
r_{4}\left(K_{2} \mathcal{O}_{E}\right)=r_{4}(C(F))+1
$$

with probability $2^{-r+1}$ as $t \rightarrow \infty$. Since $n \equiv 1 \bmod 8$ and $n \equiv 5 \bmod 8$ are equally likely in $B_{t}$, we have

$$
\gamma_{\infty, r}^{\prime}=2^{-r}
$$

By Theorem 3 of [4] and Lemma 2.1,

$$
\lim _{x \rightarrow \infty} \frac{\sum_{t=1}^{\infty}\left|B_{t, r-1 ; x}\right|}{\sum_{t=1}^{\infty}\left|B_{t ; x}\right|}=\frac{2^{-r(r-1)} \prod_{k=1}^{\infty}\left(1-2^{-k}\right)}{\prod_{k=1}^{r-1}\left(1-2^{-k}\right) \prod_{k=1}^{r}\left(1-2^{-k}\right)} .
$$

Hence

$$
\bar{d}_{r}^{\prime}(-1)=2^{-r^{2}} \frac{\prod_{k=1}^{\infty}\left(1-2^{-k}\right)}{\prod_{k=1}^{r-1}\left(1-2^{-k}\right) \prod_{k=1}^{r}\left(1-2^{-k}\right)} .
$$

TheOREM 3.2. Let $r$ be a nonnegative integer. Then the density of imaginary quadratic number fields $E$ such that $r_{4}\left(K_{2} \mathcal{O}_{E}\right)=r$ is

$$
d_{r}^{-}=2^{-r^{2}} \frac{\prod_{k=1}^{\infty}\left(1-2^{-k}\right)}{\prod_{k=1}^{r}\left(1-2^{-k}\right)^{2}} .
$$

Proof. By Theorems 3.1 and 4.1 of [16],

$$
d_{r}^{-}= \begin{cases}\bar{d}_{r}^{\prime}(0)+\bar{d}_{r}^{\prime}(1) & \text { for } r=0 \\ \bar{d}_{r}^{\prime}(-1)+\bar{d}_{r}^{\prime}(0)+\bar{d}_{r}^{\prime}(1) & \text { for } r \geq 1\end{cases}
$$

Hence we get the result by Theorem 3.1. 
4. Densities in different residue classes of $\sigma(D)$ modulo 4. In order to prove that Definition 1.1 is in accordance with Definition 1.2, we need to study the densities of real (resp. imaginary) quadratic number fields with $r_{4}\left(K_{2} \mathcal{O}_{F}\right)=r$ in the set of all real (resp. imaginary) quadratic number fields whose discriminants are in a fixed residue class modulo 8, i.e., we will study

$$
\begin{aligned}
& \sum_{0<\sigma(D)<x} g_{r}(D)
\end{aligned}
$$

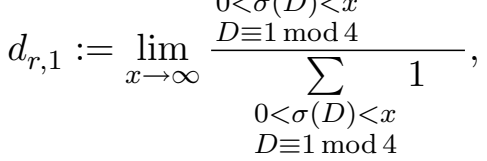

$$
\begin{aligned}
& \sum_{0<\sigma(D)<x} g_{r}(D)
\end{aligned}
$$

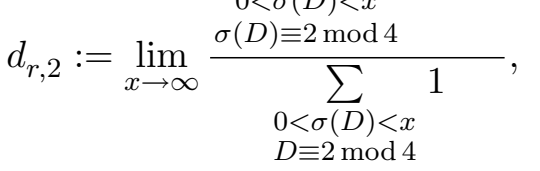

$$
\begin{aligned}
& d_{r, 2}^{\prime}:=\lim _{x \rightarrow \infty} \frac{\sum_{\substack{0<-\sigma(D)<x \\
\sigma(D) \equiv 2 \bmod 4}} g_{r}(D)}{\sum_{\substack{0(D)<x \\
0<-\sigma(D)<x \\
\sigma(D) \equiv 2 \bmod 4}} 1}, \\
& \sum_{0<\sigma(D)<x} g_{r}(D)
\end{aligned}
$$

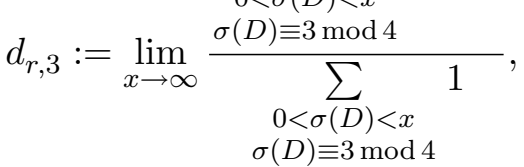

$$
\begin{aligned}
& d_{r, 1}^{\prime}:=\lim _{x \rightarrow \infty} \frac{\sum_{\substack{0<-\sigma(D)<x \\
D \equiv 1 \bmod 4}} g_{r}(D)}{\sum_{\substack{0<-\sigma(D)<x \\
D \equiv 1 \bmod 4}} 1}, \\
& d_{r, 3}^{\prime}:=\lim _{x \rightarrow \infty} \frac{\sum_{\substack{0<-\sigma(D)<x \\
\sigma(D) \equiv 3 \bmod 4}} g_{r}(D)}{\sum_{\substack{0<-\sigma(D)<x \\
\sigma(D) \equiv 3 \bmod 4}} 1} .
\end{aligned}
$$

In the proof of Theorem 2.2 in Section 2, we have used [17, (3.19)] to get $\beta_{\infty, r}$ and $\gamma_{\infty, r}$. However, equation (3.19) is proved in [17] only for $\sigma(D) \equiv$ $3 \bmod 4$. We will show that the other cases can be omitted in the proof of Theorem 2.2. Note that for any field $F=\mathbb{Q}(\sqrt{n}) \in A_{t}$, the corresponding imaginary quadratic field $E=\mathbb{Q}(\sqrt{-n})$ has exactly $t$ ramified primes. Hence either

$$
\begin{aligned}
& n=p_{1} \cdots p_{t} \quad \text { with an odd number of } p_{i} \equiv 3 \bmod 4, \text { or } \\
& n=p_{1} \cdots p_{t-1} \quad \text { with an even number of } p_{i} \equiv 3 \bmod 4 \text {, or } \\
& n=2 p_{1} \cdots p_{t-1} .
\end{aligned}
$$

Let $N_{i ; x}$ denote the number of $n<x$ satisfying (4.i) $(i=1,2,3)$. Then $N_{2 ; x}=o\left(\left|A_{t, x}\right|\right)$ and $N_{3 ; x}=o\left(\left|A_{t, x}\right|\right)$ by [5, (2.4), (2.5)]. Hence in order to get $\beta_{t, r}$ and $\gamma_{t, r}$, we need to consider only those $n$ satisfying (4.1).

Similar things happen in the proof of Theorem 3.1. However, in that proof, we confine our attention to the cases $n \equiv 1 \bmod 4$. So in Sections 2 and 3 , we have proved that

$$
\begin{aligned}
d_{r} & =d_{r, 3}, \\
d_{r}^{\prime} & =d_{r, 1}^{\prime}
\end{aligned}
$$


THEOREM 4.1. With the above notations,

(1) $d_{r}=d_{r, 1}=d_{r, 2}=d_{r, 3}$,

(2) $d_{r}^{\prime}=d_{r, 1}^{\prime}=d_{r, 2}^{\prime}=d_{r, 3}^{\prime}$.

Proof. (1) By the well known counting formulae for fundamental discriminants (cf. $[4,(16)])$,

$$
\sum_{\substack{0<\sigma(D)<x \\ D \equiv 1 \bmod 4}} 1 \sim \sum_{\substack{0<\sigma(D)<x \\ \sigma(D) \equiv 2 \bmod 4}} 1 \sim \sum_{\substack{0<\sigma(D)<x \\ \sigma(D) \equiv 3 \bmod 4}} 1 \sim \frac{2}{\pi^{2}} x \quad \text { as } x \rightarrow \infty .
$$

So $d_{r}=\left(d_{r, 1}+d_{r, 2}+d_{r, 3}\right) / 3$. Hence by (4.4), it is sufficient to prove $d_{r}=d_{r, 2}$.

Let $n$ be a square free integer, $F=\mathbb{Q}(\sqrt{n}), E=\mathbb{Q}(\sqrt{-n})$, and $A_{t}^{(2)}=\{F \mid n \equiv 2 \bmod 4$, and exactly $t$ primes ramify in $E=\mathbb{Q}(\sqrt{-n}) / \mathbb{Q}\}$, $A_{t, r}^{(2)}=\left\{F \in A_{t}^{(2)} \mid r_{4}(C(E))=r\right\}$, $A_{t ; x}^{(2)}=\left\{F \in A_{t}^{(2)} \mid n<x\right\}$, $A_{t, r ; x}^{(2)}=\left\{F \in A_{t ; x}^{(2)} \mid r_{4}(C(F))=r\right\}$, $\bar{A}_{t, r ; x}^{(2)}=\left\{F \in A_{t ; x}^{(2)} \mid r_{4}\left(K_{2} \mathcal{O}_{F}\right)=r\right\}$.

By the definition of $d_{r, 2}$, we know that

$$
d_{r, 2}=\lim _{x \rightarrow \infty} \frac{\sum_{t=1}^{\infty}\left|\bar{A}_{t, r ; x}^{(2)}\right|}{\sum_{t=1}^{\infty}\left|A_{t ; x}^{(2)}\right|} .
$$

By similar arguments to the proof of Theorem 2.2, the fields with $-1,2$ or -2 in $\operatorname{Norm}_{F / \mathbb{Q}}\left(F^{*}\right)$ can be omitted when $x$ and $t$ are both large enough.

Assume $n=p_{1} \cdots p_{t-1} p_{t}$, where $p_{1}, \ldots, p_{t-1}$ are different odd primes and $p_{t}=2$. Let $M_{E}^{\prime}$ be the $(t-1) \times t$ matrix obtained from the Rédei matrix $M_{E}$ by deleting the $t$ th row (cf. [16] and [17] for details), $Y=\left(y_{1}, \ldots, y_{t}\right)^{T}$ and $B=\left(b_{1}, \ldots, b_{t-1}\right)^{T}$, where $b_{j} \in\{0,1\}$ with $\left(\frac{2}{p_{j}}\right)=(-1)^{b_{j}}, j=1, \ldots, t-1$. Recall that $\operatorname{rank}\left(M_{E}\right)=\operatorname{rank}\left(M_{E}^{\prime}\right)$ and

$$
r_{4}(C(E))=t-1-\operatorname{rank}\left(M_{E}^{\prime}\right) .
$$

By Theorem 3.2 in [16],

$$
r_{4}\left(K_{2} \mathcal{O}_{F}\right)=r_{4}(C(E))+1
$$

if and only if the system of equations $M_{E}^{\prime} Y=B$ is solvable. Since all primes are equally distributed in the residue classes of $\pm 1, \pm 3$ modulo 8 , the probability of $r_{4}\left(K_{2} \mathcal{O}_{F}\right)=r_{4}(C(E))+1$ is $2^{-r}$ in the set $A_{t, r}^{(2)}$, as $t \rightarrow \infty$. By (4.6) and Theorem 2.2, we have

$$
d_{0,2}=\lim _{x \rightarrow \infty} \frac{\sum_{t=1}^{\infty}\left|\bar{A}_{t, 0 ; x}^{(2)} \cap A_{t, 0 ; x}^{(2)}\right|}{\sum_{t=1}^{\infty}\left|A_{t ; x}^{(2)}\right|}=0 .
$$


If $r>0$, we have

$$
d_{r, 2}=\lim _{x \rightarrow \infty} \frac{\sum_{t=1}^{\infty}\left(\left|\bar{A}_{t, r ; x}^{(2)} \cap A_{t, r+1 ; x}^{(2)}\right|+\left|\bar{A}_{t, r ; x}^{(2)} \cap A_{t, r ; x}^{(2)}\right|+\left|\bar{A}_{t, r ; x}^{(2)} \cap A_{t, r-1 ; x}^{(2)}\right|\right)}{\sum_{t=1}^{\infty}\left|A_{t ; x}^{(2)}\right|} .
$$

Since $F \in \bar{A}_{t, r ; x}^{(2)} \cap A_{t, r-1 ; x}^{(2)}$ only if $2 \in \operatorname{Norm}_{F / \mathbb{Q}}\left(F^{*}\right)$, we have

$$
\lim _{x \rightarrow \infty} \frac{\sum_{t=1}^{\infty}\left|\bar{A}_{t, r ; x}^{(2)} \cap A_{t, r+1 ; x}^{(2)}\right|}{\sum_{t=1}^{\infty}\left|A_{t ; x}^{(2)}\right|}=0 .
$$

Hence

$$
\begin{aligned}
d_{r, 2} & =\lim _{x \rightarrow \infty} \frac{\sum_{t=1}^{\infty}\left(\left|\bar{A}_{t, r ; x}^{(2)} \cap A_{t, r ; x}^{(2)}\right|+\left|\bar{A}_{t, r ; x}^{(2)} \cap A_{t, r-1 ; x}^{(2)}\right|\right)}{\sum_{t=1}^{\infty}\left|A_{t ; x}^{(2)}\right|} \\
& =\left(1-2^{-r}\right) \lim _{x \rightarrow \infty} \frac{\sum_{t=1}^{\infty}\left|A_{t, r ; x}^{(2)}\right|}{\sum_{t=1}^{\infty}\left|A_{t ; x}^{(2)}\right|}+2^{-r+1} \lim _{x \rightarrow \infty} \frac{\sum_{t=1}^{\infty}\left|A_{t, r-1 ; x}^{(2)}\right|}{\sum_{t=1}^{\infty}\left|A_{t ; x}^{(2)}\right|} \\
& =\left(1-2^{-r}\right) \frac{2^{-r^{2}} \prod_{k=1}^{\infty}\left(1-2^{-k}\right)}{\prod_{k=1}^{r}\left(1-2^{-k}\right)^{2}}+2^{-r+1} \frac{2^{-(r-1)^{2}} \prod_{k=1}^{\infty}\left(1-2^{-k}\right)}{\prod_{k=1}^{r-1}\left(1-2^{-k}\right)^{2}} \\
& =\frac{2^{-r(r-1)} \prod_{k=1}^{\infty}\left(1-2^{-k}\right)}{\left(1-2^{-r}\right) \prod_{k=1}^{r-1}\left(1-2^{-k}\right)^{2}}=d_{r} .
\end{aligned}
$$

The proof of (2) is parallel to that of (1).

From Theorem 3.1, one can easily get the following corollary.

Corollary 4.2. The densities defined in Definition 1.1 are in accordance with those in Definition 1.2.

5. The numerical evidence. In [10], Osburn and Murray, gave tables of the densities of real (resp. imaginary) quadratic number fields with $r_{4}\left(K_{2} \mathcal{O}_{F}\right)=0,1,2,3$, in the set of real (resp. imaginary) quadratic number fields $\mathbb{Q}(\sqrt{n})$ (resp. $\mathbb{Q}(\sqrt{-n})$ ) satisfying $n \leq 10^{6}$. In Table 7 of [10], the density of real quadratic number fields with $r_{4}\left(K_{2} \mathcal{O}_{F}\right)=0$ in the set of real quadratic number fields $\mathbb{Q}(\sqrt{n})$ satisfying $n<10^{6}$ is $23.1284 \%$. However, by Theorem 2.2, this density should be 0 as $n \rightarrow \infty$.

Why is the difference so large? The reason is that $10^{6}$ is not large enough. In our proof, we throw away some parts which do not affect the densities as $t \rightarrow \infty$. However, if the number of prime factors of $n$ is not large, these discarded parts in fact contribute a lot to the densities. For example, we remove all the fields satisfying $2 \in \operatorname{Norm}_{F / \mathbb{Q}}\left(F^{*}\right)$. If there are exactly two ramified primes in $F$, we have thus thrown away $25 \%$ cases, for $2 \in \operatorname{Norm}_{F / \mathbb{Q}}\left(F^{*}\right)$ if and only if every prime factor of $n$ is congruent to 1 or -1 modulo 8 . How- 
ever, the average number of prime factors of square free positive integers less than $10^{6}$ is only about 2.6 .

If one checks Table 5 of [10], one can see that the density of real quadratic number fields with $r_{4}\left(K_{2} \mathcal{O}_{F}\right)=0$ in the set of real quadratic number fields $\mathbb{Q}(\sqrt{n})$ satisfying $n=p q r \leq 10^{6}$ is only $6.827 \%$, where $p, q, r$ are different odd primes. In Tables I, II and III of [14], this density is very close to 0 if one considers those $n$ with exactly four odd prime factors.

Similar things happen for imaginary quadratic number fields. By Theorem 3.2 , this density should be about $28.88 \%$ as $n \rightarrow \infty$. However, in Table of [1], there are much more imaginary quadratic number fields $E$ with $r_{4}\left(K_{2} \mathcal{O}_{E}\right)=0$ (about $84 \%$ ) than with $r_{4}\left(K_{2} \mathcal{O}_{E}\right)>0$ in the set of imaginary quadratic number fields whose discriminant is greater than -5000 . However, if $n$ is extended to $10^{6}$, the density of imaginary quadratic number fields $E$ with $r_{4}\left(K_{2} \mathcal{O}_{E}\right)=0$ is about $62 \%$ by Table 8 in [10].

Furthermore, if one looks at the Table in [15], one can see that the density of imaginary quadratic number fields $E$ with $r_{4}\left(K_{2} \mathcal{O}_{E}\right)=0$ is apparently less than $50 \%$ in the set of imaginary quadratic number fields $\mathbb{Q}(\sqrt{-n})$ where $n$ has exactly four odd prime factors.

Acknowledgements. The author is grateful to Hourong Qin, Xiaoyun Cheng and Xiaobin Yin for helpful discussions. The main part of this paper was completed when the author was participating in the School and Conference on Algebraic $K$-theory and its Applications held at ICTP, Trieste in May 2007. The author wants to thank ICTP for hospitality.

This research was supported by NSFC 10401014, and partially supported by the Natural Science Foundation of the Jiangsu province of China (BK 2005207).

\section{References}

[1] J. Browkin and H. Gangl, Tame and wild kernels of quadratic imaginary number fields, Math. Comp. 68 (1999), 291-305.

[2] X. Cheng, A note on the 4-rank densities for $\mathrm{K}_{2} \mathrm{O}_{F}$ for quadratic number fields $\mathrm{F}$, Comm. Algebra 36 (2008), 1634-1648.

[3] P. E. Conner and J. Hurrelbrink, The 4-rank of $K_{2}(O)$, Canad. J. Math. 41 (1989), 932-960.

[4] É. Fouvry and J. Klüners, On the 4-rank of class groups of quadratic number fields, Invent. Math. 167 (2007), 455-513.

[5] F. Gerth, The 4-class ranks of quadratic fields, ibid. 77 (1984), 489-515.

[6] J. Hurrelbrink and M. Kolster, Tame kernels under relative quadratic extensions and Hilbert symbols, J. Reine Angew. Math. 499 (1998), 145-188.

[7] M. Kolster, The structure of the 2-Sylow-subgroup of $K_{2}(\mathfrak{o}) I$, Comment. Math. Helv. 61 (1986), 376-388.

[8] R. Osburn, Densities of 4-ranks of $K_{2}(\mathcal{O})$, Acta Arith. 102 (2002), 45-54. 
[9] R. Osburn, A note on 4-rank densities, Canad. Math. Bull. 47 (2004), 431-438.

[10] R. Osburn and B. Murray, Tame kernels and further 4-rank densities, J. Number Theory 98 (2003), 390-406.

[11] H. Qin, The 4-rank of $\mathrm{K}_{2} \mathrm{O}_{F}$ for real quadratic fields $F$, Acta Arith. 72 (1995), $323-333$.

[12] - The 2-Sylow subgroups of the tame kernel of imaginary quadratic fields, ibid. 69 (1995), 153-169.

[13] - , Tame kernels and Tate kernels of quadratic number fields, J. Reine Angew. Math. 530 (2001), 105-144.

[14] X. Yin, The 4-rank of tame kernels for real quadratic fields F, preprint.

[15] - The 4-rank of tame kernels for imaginary quadratic fields F, preprint.

[16] Q. Yue and K. Feng, The 4-rank of the tame kernel versus the 4-rank of the narrow class group in quadratic number fields, Acta Arith. 96 (2000), 155-165.

[17] Q. Yue and J. Yu, The densities of 4-ranks of tame kernels for quadratic fields, J. Reine Angew. Math. 567 (2004), 151-173.

Department of Mathematics

Nanjing University

Nanjing 210093, China

E-mail: guoxj@nju.edu.cn 